\title{
Prevalence and Pattern of Craniofacial Pain and Headache in Danish Patients with Neuromyelitis Optica Spectrum Disorder
}

\author{
Gebbie Ann Rodriguez Nielsen, ${ }^{1}$ Helle Hvilsted Nielsen, ${ }^{2}$ Zsolt Laszlo Illés² and Parisa Gazerani ${ }^{1}$ \\ 1. Department of Health Science and Technology, Faculty of Medicine, Aalborg University, Aalborg, Denmark, \\ 2. Department of Neurology, Odense University Hospital, University of Southern Denmark, Odense, Denmark
}

DOI: https://doi.org/10.17925/ENR.2018.13.1.44

$\mathrm{T}$ here is a dearth of knowledge about the pattern of pain and somatosensory alterations that co-exist with neuromyelitis optica spectrum disorder (NMOSD). Understanding the presence and underlying mechanisms will assist in better pain management in these patients. This study sought to identify prevalence and pattern of headache, craniofacial pain and abnormalities in somatosensory function within a group of NMOSD patients in Denmark. Six patients (two males, four females; mean age $41.2 \pm 8.6$ years) participated in a telephone interview. Eight questionnaires were used to assess pain, illness perception, fatigue, anxiety and depression. Pain occurred in several regions, including the head, face, neck, back and legs. Two patients experienced frequent headaches and one had headaches following neck pain. Pain occurred frequently over a 3-month period, ranging from mild to severe and described as 'burning' (66.7\%), 'exhausting' (83.3\%) and 'sharp' (50\%). Correlations were found between pain severity and patients' 'ability to walk' $(r=0.889)$, 'general activity' ( $r=0.901)$, 'mood' $(r=0.603)$, 'normal work' ( $r=0.664)$, 'relations with other people' $(r=0.774)$ and 'sleep' $(r=0.586)$. Somatosensory abnormality was only reported in legs. While fatigue had a great impact on patients' daily life, patients did not report that for anxiety and depression. This study demonstrated that headaches and craniofacial pain occur frequently in NMOSD. Patients' function, mobility, mood and sleep were all affected by pain. These findings highlight the value of further investigation on headaches, craniofacial and overall pain in NMOSD.

\section{Keywords}

Pain, craniofacial, headache, neuromyelitis optica spectrum disorder, NMOSD, Danish

Disclosure: Gebbie Ann Rodriguez Nielsen, Helle Hvilsted Nielsen, Zsolt Laszlo Illés and Parisa Gazeran have nothing to declare in relation to this article. Review Process: Double-blind peer review

Compliance with Ethics: All procedures were followed in accordance with the responsible committee on human experimentation and with the Helsinki Declaration of 1975 and subsequent revisions.

Authorship: All named authors meet the International Committee of Medical Journal Editors (ICMJE) criteria for authorship of this manuscript, take responsibility for the integrity of the work as a whole, and have given final approval to the version to be published.

open Access: This article is published under the Creative Commons Attribution Noncommercial License, which permits any non-commercial use, distribution, adaptation and reproduction provided the original authors and source are given appropriate credit. @ The Authors 2018.

Received: 8 February 2018

Accepted: 12 March 2018

Citation: European Neurological

Review. 2018;13(1):44-52

Corresponding Author: Parisa Gazerani,

Department of Health Science and Technology,

Aalborg University, Frederik Bajers Vej 7A2-A2-208,

9220 Aalborg East, Denmark. E: gazerani@hst.aau.dk

LinkedIn: linkedin.com/in/parisa-gazerani-2297122/

Support: No funding was received for conduction of this study or preparation of this article.

\section{Neuromyelitis optica spectrum disorder}

Neuromyelitis optica spectrum disorder (NMOSD) is an autoimmune inflammatory disease of the central nervous system (CNS) causing severe inflammation and demyelination of the optic nerve (optic neuritis [ON]), the spinal cord (transverse myelitis [TM]) and the brainstem. ${ }^{1.2}$ There is a dearth of epidemiological data on the prevalence of NMOSD; conclusive data on the occurrence of NMOSD are lacking and worldwide there are few epidemiological studies on the disorder. ${ }^{3-5}$ It has been estimated that the prevalence of NMOSD ranges from 1.0-4.4 out of 100,000 in the Western world (Europe and North America). ${ }^{3.6}$ Strikingly, more women are diagnosed with NMOSD than men (ratio 9:1). ${ }^{3}$ The typical age of disease onset peaks at approximately 35-45 years, however, it also has been observed in children and elderly. ${ }^{6}$ The common clinical presentation of NMOSD consists of severe episodes of simultaneously bilateral $\mathrm{ON}$, which in turn causes visual field defect or loss of vision. ${ }^{7-9}$ Spinal cord symptoms range from mild sensory disturbances to complete TM, causing tetraplegia, paraplegia or paralysis accompanied by sensory impairments and bladder/ bowel dysfunction. ${ }^{-9}$ Brainstem symptoms consist of intractable hiccups or nausea and vomiting, also known as area postrema syndrome. ${ }^{8.9}$ Attacks of ON or myelitis are highly debilitating and if left untreated, the prospect of remission would be poor, which as a result will lead to irreversible neurological damage and disability. ${ }^{6}$ In addition to the common core symptoms, pain syndromes happen to occur more frequently than first appreciated; however, the underlying mechanisms causing these pain conditions in NMOSD have not been fully elucidated. ${ }^{1.6}$ Despite a large variety of pain medications, of which each targets different mechanisms of pain, and the different combinations of these medications, NMOSD-associated neuropathic pain is yet unmanageable. In turn, the everyday life of patients is affected to an extent that significantly lowers their quality of life.? A study in 2016 has demonstrated that depression in NMOSD patients is linked to neuropathic pain and fatigue if these conditions are poorly treated..$^{10}$ Better knowledge regarding the mechanisms of pain in NMOSD patients is urgently needed in order to expand existing knowledge, which in the long run could be beneficial for this group of patients.?

More than $85 \%$ of NMOSD patients have reported pain as a major symptom and that, despite the frequent use of pain medication (e.g. neuropathic medications, antiepileptic medications, opioids and non-opioids), they unceasingly experienced high pain intensities. ${ }^{11}$ Painful tonic muscle spasms and neuropathic pain are the most common pain types in NMOSD, causing burning, sharp, electrical, shooting pain, cramps, icy or scalding sensations, or numbness. ${ }^{12}$ These types of pain highly interfere with the quality of life of NMOSD patients. ${ }^{11,1,12}$ While ON typically causes pain around or behind 
Figure 1: Study overview representing the setup and steps taken for the telephone interview

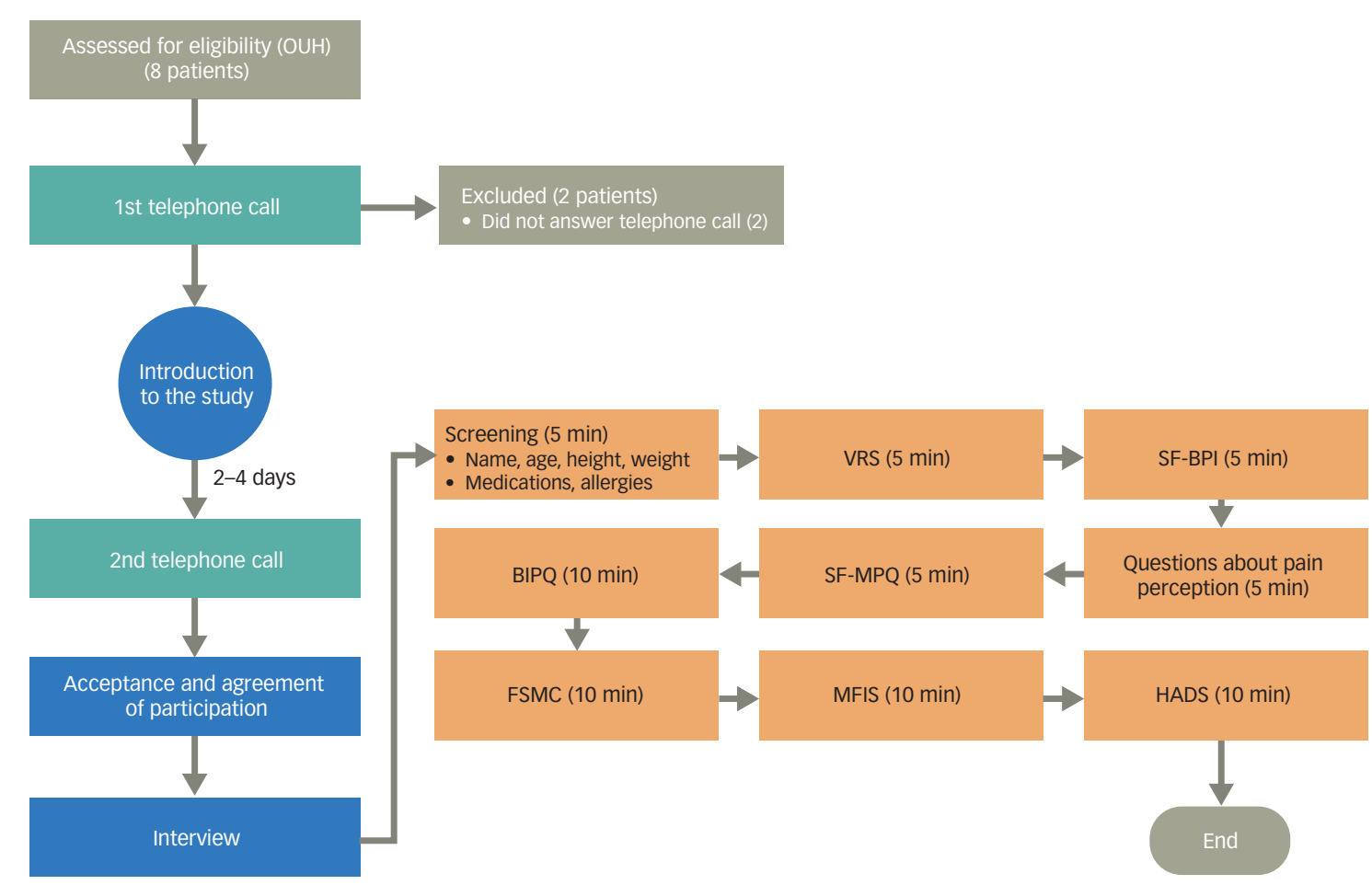

Patients were recruited from the Department of Neurology at Odense University Hospital (OUH). All patients were contacted by phone and those not answering were not included. Prior to the actual interview, patients were introduced to the study purpose and were given 2-4 days consideration to participate. Once they accepted and agreed to participate, the actual interview was conducted. BIPQ = Brief IIIness Perception Questionnaire; FSMC = Fatigue Scale for Motor and Cognitive Functions; HADS = Hospital Scale for Anxiety and Depression; MFIS = Modified Fatigue Impact Scale; OUH = Odense University Hospital; SF-BPI = Short-form Brief Pain Inventory; SF-MPQ = Short-form McGill Pain Questionnaire; VRS = Verbal Rating Scale.

the eyes and TM causes chest and back pain radiating into the lower extremities and motor and sensory problems, data regarding the pattern of pain in the craniofacial regions and the prevalence of headache in NMOSD has not been reported..$^{1,11-13}$ Moreover, hyper- and hypophenomena of pain (e.g. hyperalgesia, allodynia and paradoxical heat sensations) have primarily been reported in areas such as the hand, foot and extremities, while knowledge of the pattern of somatosensory changes in the craniofacial regions of the NMOSD patients still largely remain limited or missing. ${ }^{12}$ This accentuates the necessity of further investigating patterns of pain and somatosensory function in NMOSD ${ }^{14}$ to identify the underlying mechanisms of pain in NMOSD and gain better control.

\section{Study aim}

This study aimed to identify the prevalence and pattern of pain, and abnormalities of somatosensory function in NMOSD patients, by examining sensory hyper- and hypophenomena through a panel of questionnaires. Pain patterns in the craniofacial region and headaches have rarely been investigated in NMOSD, and this study attempts to shed light on this neglected feature. In addition to pain, fatigue and depression are common comorbidities in NMOSD, ${ }^{15,16}$ which highly affect patients' quality of life and may worsen their pain experience. Consequently, this study sought to explore the existence and pattern of these conditions through questionnaires addressing pain, fatigue and depression.

\section{Methods}

\section{Study design and experimental setup}

The study protocol was communicated with the regional ethics committee of the central region in Denmark for obtaining ethical approval, but it was confirmed that there was no necessity for an approval on conduction of this qualitative study. All patients participated in a phone interview voluntarily and it was explained to them that they could withdraw at any time and refuse to answer questions without any explanations or any consequences.

\section{Patients}

Patients were recruited through the Department of Neurology at Odense University Hospital (OUH), Denmark. Patients were eligible to take part in this study if they had been diagnosed with NMOSD according to the 2015 diagnostic NMOSD criteria, ${ }^{8}$ were both AQP4 positive and negative and aged 18 years or above. Patients were contacted by the investigator by telephone a few days before the actual interview and were given a short introduction regarding the purpose of the study to allow them to consider their participation. Each patient had at least 2 days to reflect upon participating before the next contact was made. Patients were asked whether they still wished to participate and, in case of acceptance and agreement, the actual telephone interview was conducted at an agreed date and time. To ensure that all information regarding the study, descriptions of questionnaires and questions from the questionnaires were similar for each patient interview, thus ensuring homogeneity for later analysis, the investigator had a manuscript on hand. The interview consisted of eight questionnaires and was designed to take up to one hour at the longest, as depicted in Figure 1. Patients were excluded from the interview if they withdrew or could not cooperate.

\section{Interviews}

Patients were interviewed by telephone due to the distance between the investigator and the patients. Furthermore, the telephone interviews were the most convenient way of interviewing patients with vision and/ 
or mobility disabilities. The investigator (GARN) presented herself as a researcher and made it clear that she did not have the qualifications or experience to make a medical diagnosis nor treat patients, nor did she have a specific allegiance to the Department of Neurology at OUH.

All questionnaires used for the interview addressed the common comorbidities of NMOSD such as pain (including sensory function and dysfunction), fatigue and depression. A Danish translation of the Short-form Brief Pain Inventory (SF-BPI) and the Short-form McGill Pain Questionnaire (SF-MPQ) were used to address pain prevalence, localisation on the body, intensity and influence on the everyday life of the patient. The Brief IIness Perception Questionnaire (BIPQ), Fatigue Scale for Motor and Cognitive Functions (FSMC), Modified Fatigue Impact Scale (MFIS), Beck Depression Inventory Fast Screen (BDI-FS) and the Hospital Scale for Anxiety and Depression (HADS) were used to address fatigue, anxiety and depression, and how it affects the quality of life of the patient. In addition to the questionnaires, patients were asked to rate their pain experiences with a Verbal Rating Scale (VRS). Patients were questioned regarding their pain status in a given body part: their current pain, pain during the last week, during the last month, and during the last three months. Furthermore, a brief questionnaire regarding the patient's pain perception was used. This questionnaire was specifically designed to address hypo- or hyperphenoma of pain, based on questions like 'Does light touch (e.g. with clothing, bed-linen, blankets etc.) intensify pain in areas of pain?'. The interview followed a structured interview guide, ensuring that all questions were relevant for all patients and to retrieve relevant information, however, patients were also given the possibility to address issues that were important for them to share. Interviews were not audio-recorded and transcribed. All answers to questionnaires, additional questions, as well as other related information were reported in a case record form during the actual interview.

\section{Statistical analysis}

Data collection was made in Excel and analyses were conducted using the software SPSS Statistics (version 24, IBM, Armonk, NY, US) with a statistical significance level at $p<0.05$.

The VRS consisted of a four-point scale with the words 'no pain', 'slight pain', 'moderate pain' and 'severe pain'. Patients were questioned regarding their pain status (i.e. their current pain, pain during the last week, during the last month and during the last three months) in four different body areas: (1) face, head and neck; (2) shoulders, arms and hands; (3) chest, stomach and upper/lower back; and, lastly (4), legs and feet. Data were analysed with a frequency analysis.

The brief questionnaire about pain and its quality was assessed with a six-point scale with the words 'never', 'almost never', 'sometimes', 'often', 'almost always' and 'always'. Data were analysed using frequency analysis. Localisation of pain was reported and sketched on illustrations of the anterior and posterior surfaces of the body (body-charts), and were then superimposed with use of appropriate transparencies using a computer-drawing program (Adobe Photoshop CC 2017, Adobe, San Jose, CA, US). Characterisation of pain was analysed using frequency analysis.

The SF-BPI consisted of a series of numerical rating scales (NRS) $(0=$ no pain to $10=$ worst imaginable pain) to address pain (current pain status as well as worst and mildest pain in the past 24 hours) (pain severity indices). Additionally, the pain-related interferences, i.e. how pain had affected patients for the past 24 hours, was addressed on a NRS $(0=$ no interference and $10=$ complete interference). A mean NRS for each NRS- series was calculated and assessed. A Pearson's correlation analysis was computed for the pain severity indices and pain-related interferences. The SF-MPQ assesses the characteristics of pain with a series of pain descriptors. A frequency analysis was used to assess the amount of chosen descriptors.

The BIPQ is a nine-item questionnaire used to measure illness perception. Each item was answered with the help of an 11-point scale (0-10). Each of the items represented different dimensions (identity, timeline, consequence, personal control, treatment control, concern understanding and emotional representation), and higher scores indicate stronger endorsement of that item. All scores were added up to reflect the overall positivity or negativity of an individual's illness perceptions. The minimum score was 0 and the maximum score was 90.

FSMC and MFIS were used to assess fatigue with five-point scales (1-5 for FSMC and 0-4 for MFIS). Then, all scores were compiled. The FSMC scores were further divided into two subscales: a mental and a fatigue subscale. All items were scaled so that higher scores indicated a greater impact of fatigue on a patient's activities

BDI-FS and HADS were used to address depression using four-point scales (0-3). For data obtained from BDI-FS, the total score from each item was calculated and used to determine severity of depression, where high total scores indicate more severe depressive symptoms. Cut-off scores used were as follows: 0-9 indicates minimal depression, 10-18 indicates mild depression, 19-29 indicates moderate depression, and 30-63 indicates severe depression. Questions from the HADS questionnaire were divided into two subscales: depression and anxiety. A minimum score for each of the two subscales is 0 , while a maximum score is 21. Cut-off scores were: $0-7$ is 'normal' (indicates no anxiety or depression), 8-10 is 'borderline abnormal' (indicates some anxiety or depression), and 11-21 is 'abnormal' (indicates anxiety or depression).

\section{Results}

Six NMOSD patients (four females, two males), with an average age of $41.2 \pm 8.6$ years (mean \pm standard error of the mean [SE]) and a BMI of $24.2 \pm 0.9 \mathrm{~kg} / \mathrm{m}^{2}$ (mean $\pm \mathrm{SE}$ ), participated voluntarily in this study. Two out of six patients did not take pain medication on the interview day. Eight patients were initially contacted; however, two patients were not included in the interviews, since they did not respond to the telephone calls. No complication occurred during the interview and no complaint was raised. Patient demographic information is detailed in Table 1.

\section{Pain}

Pain was assessed through application of different questionnaires: VRS, SF-BPI, SF-MPQ and a brief questionnaire regarding pain. Most of the questionnaires share identical questions. To avoid redundancy in the interview, potential confusion and to save time, the investigator only asked patients about pain characteristics once, rather than, for example, three times in three different questionnaires.

\section{Distribution of pain - verbal rating scale (VRS) and body charts}

Table 2 shows the ratings from the VRS from different body parts over time. Overall, the pain intensity - regardless of region - varied from patient to patient and was reported to range from mild to severe. Findings from distribution of pain showed that, for nearly half of all patients, pain seemed to occur in either the head-face-neck region, torso, legs or in all of the three areas. Pain was not present in the arms, shoulders or hands in this population as no patient reported pain in any of those regions. For 
Table 1: Patient demographics

\begin{tabular}{|c|c|c|c|c|c|c|c|}
\hline Patient & $\begin{array}{l}\text { Age } \\
\text { (years) }\end{array}$ & Gender & $\begin{array}{l}\text { Height } \\
(\mathrm{cm})\end{array}$ & $\begin{array}{l}\text { Weight } \\
(\mathrm{kg})\end{array}$ & $\begin{array}{l}\text { BMI } \\
\left(\mathrm{kg} / \mathrm{m}^{2}\right)\end{array}$ & Medications & Other disorders \\
\hline 1 & 40 & Female & 164 & 70 & 26.03 & Contraceptives, acetaminophen, ibuprofen & \\
\hline 2 & 35 & Female & 173 & 66 & 22.05 & Fish oil, vitamins, vitamin D, calcium, azathioprine, Benadryl ${ }^{\circledR}$ & Pollen allergy \\
\hline 3 & 68 & Female & 177 & 70 & 22.34 & $\begin{array}{l}\text { Baclofen, sertralin, omeprazol, Betmiga (mirabegron), Trimopan }{ }^{\circledast} \\
\text { (trimethoprim) }\end{array}$ & Penicillin allergy \\
\hline 5 & 25 & Female & 180 & 71 & 21.91 & Contraceptives (Femicept ${ }^{\circledR}$ ), fish oil, vitamins (Longovital ${ }^{\circledR}$ ) & Allergy, atopic dermatitis \\
\hline 6 & 36 & Male & 196 & 103 & 26.10 & Magnesium, cannabis, $\mathrm{Klyx}^{\circledast}$, vitamin D3, atorvastatin, baclofen & \\
\hline
\end{tabular}

$B M I=$ body mass index.

Table 2: Summary of verbal rating scale findings

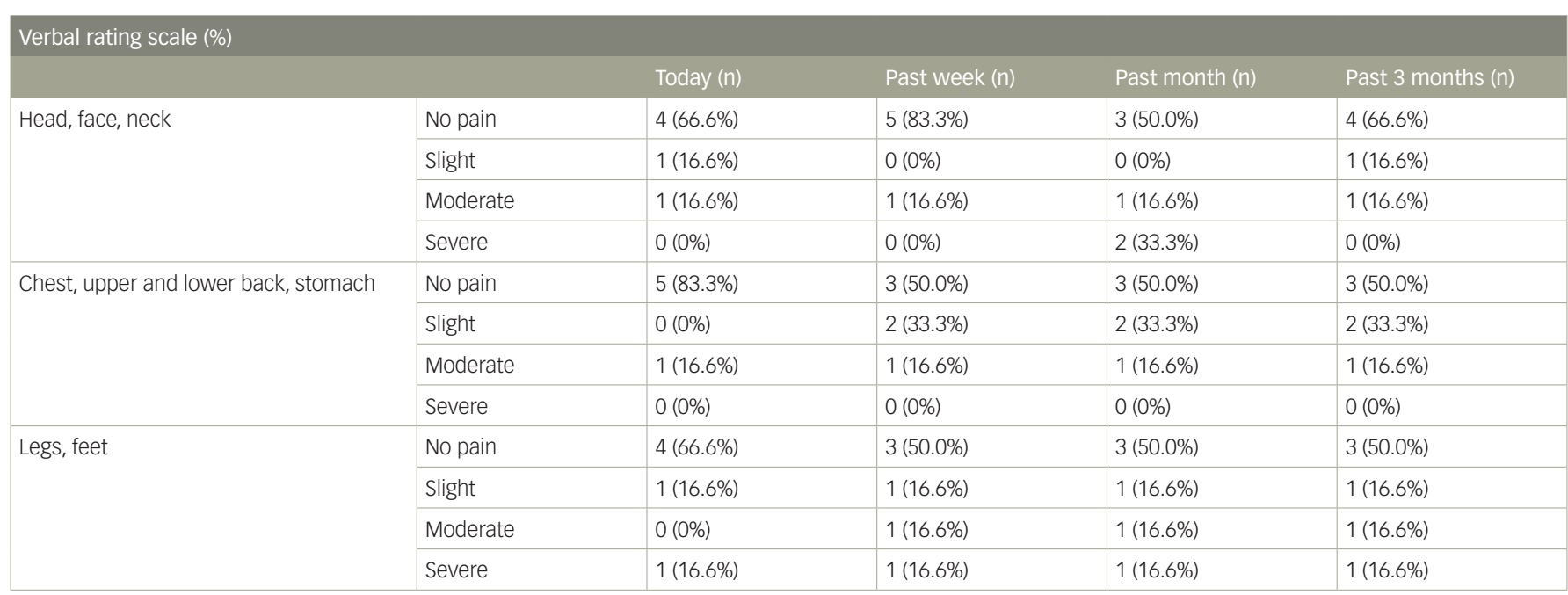

Patients were asked to rate their pain over the past 3 months in four different regions of the body: head, face and neck; shoulders, arms and hand (data not shown); chest, upper/lower back and stomach; and legs and feet. Results are presented as the number (percentage) (n [\%]) of patients rating any of the four rating options: 'no pain', 'slight pain', 'moderate pain' and 'severe pain'. Total number of patients $=6$.

the head-face-neck region, two patients reported periodic occurrence of headaches, while one patient reported constant neck pain, which could become headache if untreated. Two of the patients, one with the periodic headaches and the other with neck pain, also reported that physical exertion could trigger pain in and around the orbital region, however, it had occurred over three months ago. For the chest-upper/lower backstomach region, two patients reported pain in the lower back (one constantly occurring, one periodic), while one patient experienced almost constant pain in both upper and lower back. Two of these patients also reported some pain in or around the stomach area (tightening pain). For the leg-feet region, three patients reported pain in the legs (two constant, one almost constant). One of the three also reported severe constant pain in the feet. The areas of frequently occurring pain in patients are depicted in Figure 2. Pain was reported to occur mostly in areas of the head, neck, upper back, lower back and legs. One patient, marking the area of the neck and shoulders, reported that the pain did not involve the shoulders but appeared on the entire upper back. Another patient reported pain on the plantar surface of the feet.

\section{Short-form Brief Pain Inventory (SF-BPI)}

The pain severity index from the SF-BPI is illustrated in Figure 3. The graph shows the mean score of pain for the past 24 hours when the pain was at the highest or lowest, the pain on average, and current pain. The mean pain severity indices show that patients experience mild (1-3) to moderate (4-6) pain over the past 24 hours. Table 3 shows mean painrelated interferences. The pain-related interferences consist of seven domains (general activity, mood, walking ability, normal work, relations with other people, sleep and enjoyment of life), which patients were asked to rate on a scale from 0-10, where $0=$ no interference and $10=$ complete interference, how much their pain affected these domains. The results show that pain - to some degree - affects patients in each of the seven domains. A Pearson correlation analysis was conducted to determine the relationship between the pain severity indices (average) and the pain-related interferences (see Table 3) Results show that a strong positive correlation was present for the pain severity index and 'ability to walk' ( $r=0.889, n=5, p=0.044)$ and 'general activity' $(r=0.901, n=6$, $\mathrm{p}=0.014)$. Furthermore, a strong correlation was observed between the average pain severity index and pain-related interferences 'mood', 'normal work', 'relations with other people', and 'sleep', although these were not significant.

\section{Short-form McGill Pain Questionaire (SF-MPQ)}

Patients were asked to select pain descriptors that described their pain experiences the best. The most frequently chosen descriptors were: 'throbbing' (66.7\%), 'shooting' (50\%), 'pricking', 'sharp' (50\%), 'cramping' (50\%), 'pulling' (50\%), 'burning' (66.7\%), 'tender' (66.7\%), 
Figure 2: Superimposed illustration of frequently occurring painful areas

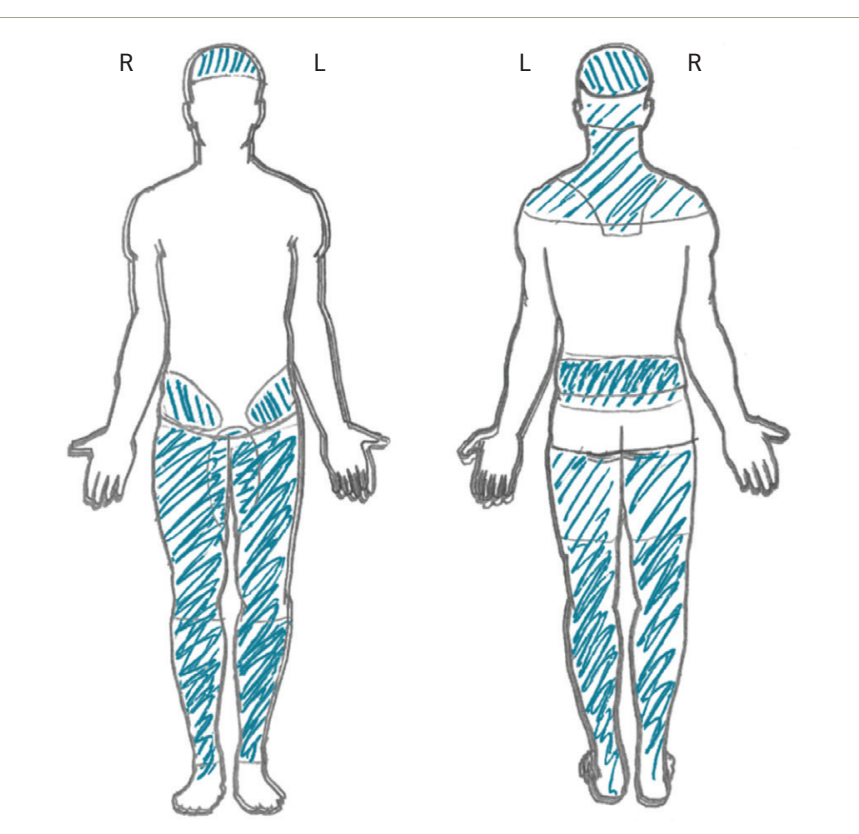

Patients were asked to specifically describe where the pain occurred. The investigator marked out areas according to patient descriptions. One patient reported pain under the foot; this could not be sketched on the illustration. Patients reported frequently occurring pain in areas of head, neck, upper back, lower back, and legs. Total number of patients $=6 . \mathrm{L}=$ left; $R=$ right.

'exhausting' (83.3\%), and 'annoying' (83.3\%). The results from the SFMPQ are illustrated in Figure 4, which shows the number of selected pain descriptors.

\section{Brief questionnaire about pain}

The brief questionnaire about pain was made for the sole purpose of investigating the quality of pain, and to identify if hypo- and/or hyperphenomena of pain exist and to what extent. Results showed that everyday stimuli such as light touch (e.g. clothing, bed linens, blankets, etc.), pressure (e.g. with a finger), and coldness and/or warmth (e.g. bathing) could trigger pain in 3-4 patients. Two of these patients reported that this occurred 'always', while the remaining patients reported that it occurred 'almost never' and 'slightly'. None of these patients reported that such phenomena occurred for the head, face or neck. However, it was reported that such phenomena commonly occurred for the areas of the lower back and legs. Only one patient experienced pain attacks and a numb sensation in lower back and legs and that this occurred 'always'.

\section{Illness perception}

Results from the BIPQ showed a mean total score of 38.7 arbitrary units (a.u.) \pm 9.5 (Table 4). This indicates that the patients - to some degree think negatively about their disease. In particular, item 2 (timeline: 'how long do you think you will be sick?') rated highest (9.0 a.u. \pm 0.6 ), indicating that patients believed that the prospect of recovery is poor. The causal item, i.e. item 9, consisted of the patients' own thoughts about what may have caused their diagnosis. Most patients did not know why and could not give their own ideas to causes of their illness. However, those who did answer were somewhat unanimous in their answers: their disease was stress-induced or caused by lifestyle factors, e.g., smoking. One patient reported that genetic predisposition (autoimmunity runs in the family) may play a role. Another patient reported that prior infections may have caused the disease.
Figure 3: Mean pain severity indices

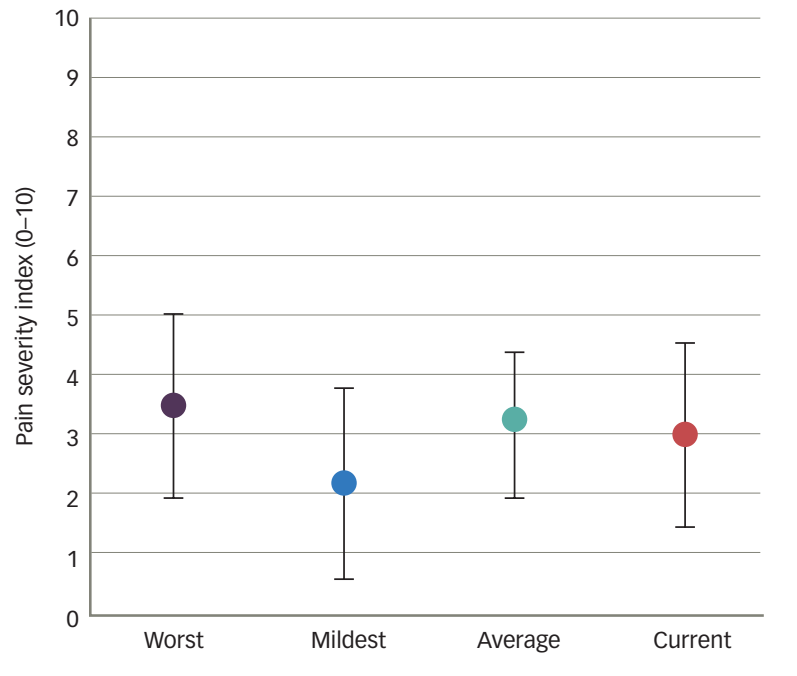

The investigator asked the patients to rate their pain with a scale ranging from 0-10 $(0=$ no pain, $10=$ worst imaginable pain) over a time period of 24 hours. Patients were asked to rate the severity of their pain when it was worst and mildest, what it was on average, and their current pain status. Mean pain scores were subcategorised into no pain (0), mild pain (1-3), moderate pain (4-6) and severe pain (7-10). Error bars $\pm S E$. Total number of patients $=6 . S E=$ standard error of the mean.

\section{Fatigue}

Similar to the questionnaires of pain, many questions were identical for the fatigue questionnaires. As a specific scoring system was used to analyse these questionnaires, identical questions were repeated. To avoid confusion, the investigator explained to patients that they might have to answer identical questions in different questionnaires. They were also instructed to not think too much about their answer, however, they should answer what they felt about their situation. Scores from one patient were not obtained due to discontinuation of the interview.

\section{Fatigue Scale for Motor and Cognitive Functions (FSMC) and Modified Fatigue Impact Scale (MFIS)}

The mean total score of the FSMC was calculated, which showed a mean score of 50.8 (a.u.) \pm 7 .3. This indicates that the patients suffer from fatigue as a result of their disease. The score was divided into two subscales: a mental and a fatigue subscale. The mental subscale had a mean score of 25.5 (a.u.) \pm 4.5 , while the fatigue subscale had a mean score of 25.3 (a.u.) \pm 3.6 , indicating that mental fatigue and physical fatigue equally contributed to an overall fatigue score in the patients. The MFIS is a questionnaire assessing fatigue over a period of 4 weeks. A mean total MFIS score was calculated, which showed a mean score of 23.0 (a.u.) \pm 6.1 , indicating that fatigue had had an impact on the patient's everyday life for the past 4 weeks.

\section{Depression and anxiety}

The questionnaires of depression and anxiety overlapped to some extent, similar to the questionnaires of pain and fatigue. Patients were asked to answer the questions regardless of their repetition. This was done as each answer represents a certain score, which would be used in a specific scoring system. Scores from one patient were not obtained due to discontinuation of the interview.

\section{Beck Depression Inventory Fast Screen (BDI-FS) and Hospital Scale for Anxiety and Depression (HADS)}

The results from the BDI-FS showed a total mean score of $1.6 \pm 0.8$, which indicates that the patients suffer from minimal depression. 
Table 3: Scores for mean pain-related interferences and Pearson's correlation analysis

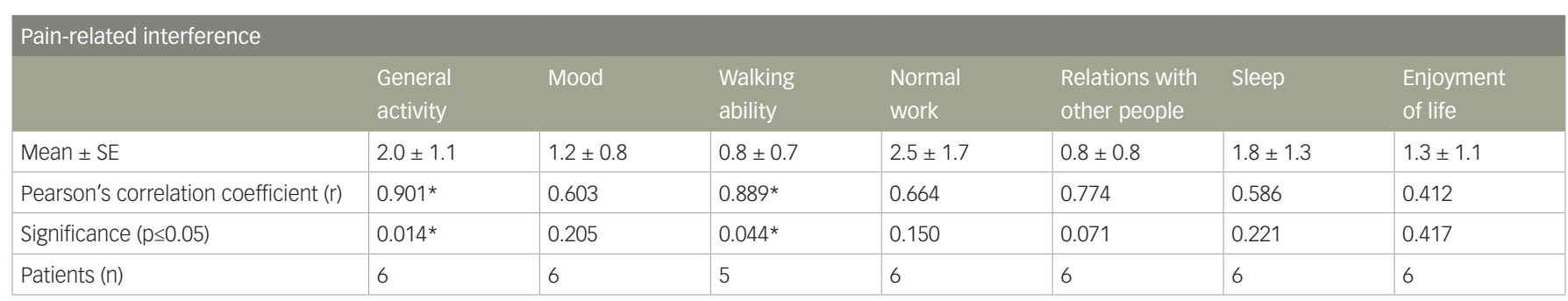

Mean pain-related interferences were rated on a scale ranging from 0-10 ( $0=$ not at all, $10=$ completely). Correlation between the pain severity index (average) and the painrelated interferences revealed a strong correlation for pain severity index and 'general activity', 'walking ability', 'mood', 'normal work', 'relations with other people', and 'sleep'. A Pearson correlation coefficient of 0.5-1.0 indicates a strong correlation, while a coefficient of $0.3-0.5$ indicates moderate correlation and, lastly, 0.1-0.3 indicates low correlation. Only 5 patients rated in the item 'walking ability', as one patient was paralysed from chest to feet. Significant values are indicated with an asterisk (*). Total number of patients =

5-6. SE = standard error of the mean.

Table 4: Mean Brief Illness Perception Questionnaire scores $( \pm$ SE) for each item of the questionnaire and a total mean score

\begin{tabular}{|c|c|c|c|c|c|c|c|c|}
\hline \multicolumn{9}{|c|}{ Mean BIPQ scores (a.u.) \pm SE } \\
\hline Consequence & Timeline & $\begin{array}{l}\text { Personal } \\
\text { control }\end{array}$ & $\begin{array}{l}\text { Treatment } \\
\text { control }\end{array}$ & Identity & Coherence & $\begin{array}{l}\text { Emotional } \\
\text { representation }\end{array}$ & $\begin{array}{l}\text { Concern } \\
\text { understanding }\end{array}$ & Total score \\
\hline $5.5 \pm 1.4$ & $9.0 \pm 0.6$ & $3.8 \pm 2.0$ & $3.8 \pm 2.0$ & $4.5 \pm 1.1$ & $4.7 \pm 1.4$ & $3.8 \pm 1.7$ & $4.7 \pm 1.5$ & $38.7 \pm 9.5$ \\
\hline
\end{tabular}

Patients were asked to answer each item with the use of a scale ranging from 0-10. The higher the rating, the stronger the endorsement of that item. Each item represents different dimensions (consequences, timeline, personal control, treatment control, identity, coherence, emotional representation, concern understanding and, lastly, the causal item). The last item (causal item) is not shown in the table. Total number of patients = 6. a.u. = arbitrary units; BIPQ = Brief IIIness Perception Questionnaire; SE: standard error of the mean.

Figure 4: Bar chart of number of chosen pain descriptors from the Short-form McGill Pain Questionnaire

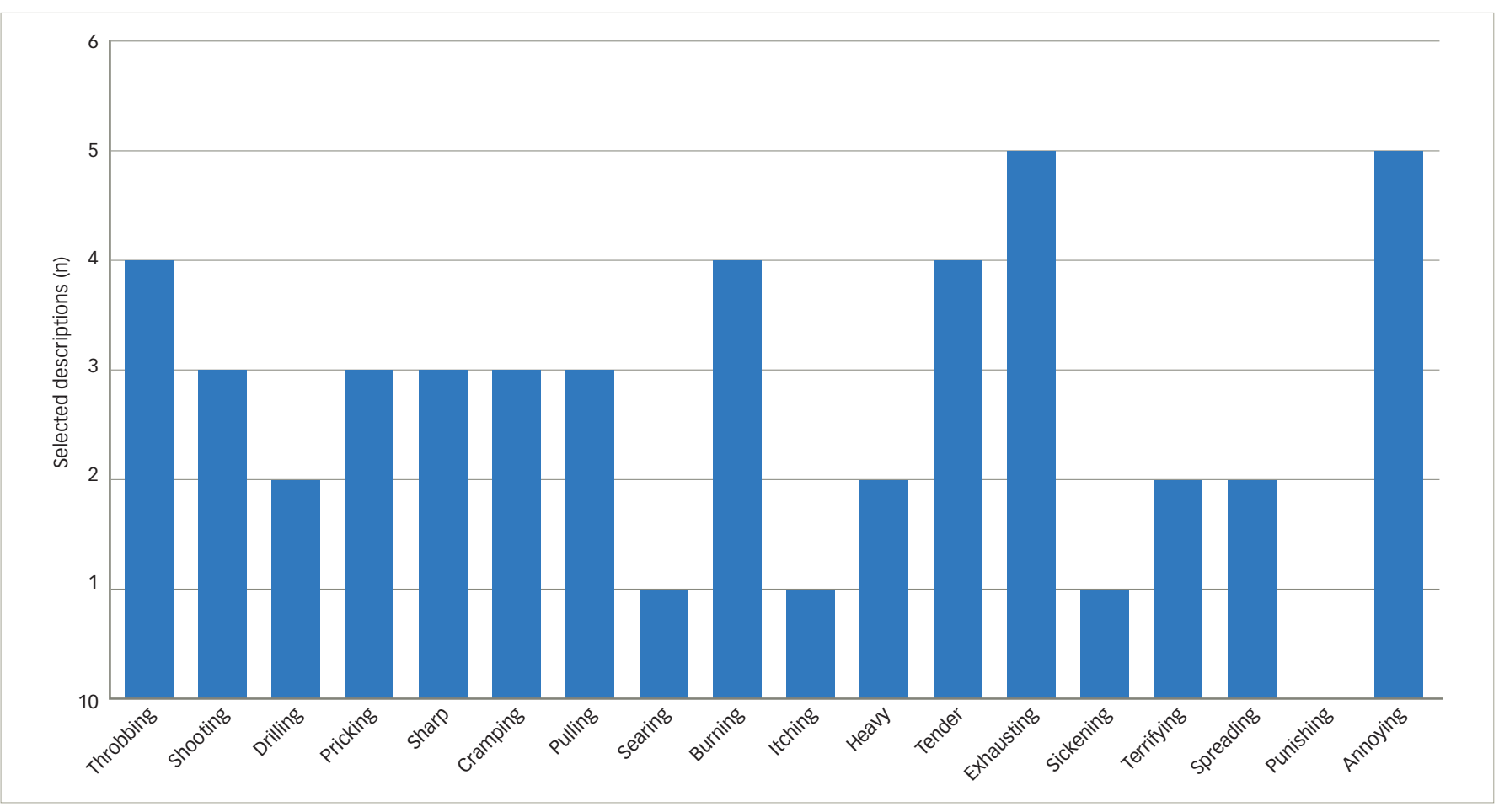

Patients were asked to choose the best descriptors to reflect on their pain experiences. Total number of patients $=6$.

The HADS questionnaire is divided into an anxiety subscale and a depression subscale. Results from the HADS showed a mean score of 4.4 (a.u.) \pm 2.1 for the anxiety dimension indicating no presence of anxiety, while a mean score of 1.6 (a.u.) \pm 0.8 for the depression dimension indicates no depression. However, one patient scored as high as 12 on the anxiety subscale, indicating a high degree of anxiety.

\section{Discussion}

The present study investigated the prevalence and pattern of pain and abnormalities of somatosensory function in NMOSD patients, with additional attention to craniofacial pain and headaches. The impact of fatigue, anxiety and depression on pain experiences in these patients was also studied. Through a series of questionnaires, this study demonstrated 
that pain was present in these patients and that in half of them pain seems to dominate in either the head-face-neck region, back, legs, or in all three areas. For the back-region and legs, patients experienced mild to severe pain. Two patients experienced frequently occurring headaches and one had headaches following neck pain. This occurred frequently over a three-month period and was described as ranging from mild to severe. No alteration was reported in terms of somatosensory function within the craniofacial region, but hypo- or hyperphenomena were present in legs. While anxiety and depression appear not to affect this group of patients, the results show that fatigue plays a great role in their everyday life. The inter-relationship between fatigue, pain, anxiety and depression has been found for many pain conditions $S^{4,17,18}$ and these findings call for further investigation of the fatigue-pain relationship in these patients.

\section{Overall pain experience}

Pain is a prevalent symptom in NMOSD and it has been reported that a majority of patients suffering from this disease complain about constant pain. ${ }^{18}$ This was also the case for the patients interviewed in this study. When reviewing the results from the VRS and SF-BPI, pain was found to be at least more or less constant within the last three months, and even on the day of the interview patients experienced mild to moderate pain. In general, pain seemed to occur in the headface-neck region, torso and legs; however, it was present in the legs and in the lower back predominantly. This is in agreement with existing studies reporting that pain is most prevalent in legs (89\%) and back (93\%). ${ }^{1,11-13}$ These studies also state that 'burning', 'exhausting' and 'sharp' are used frequently to describe pain characteristics in these patients. Similarly, the results from the SF-MPQ used in the present study showed that 'burning' (66.7\%), 'exhausting' (83.3\%) and 'sharp' (50\%) were also the most frequently chosen descriptors. In addition to that, often-chosen descriptors were also 'throbbing' (66.7\%), 'shooting' (50\%), 'pricking' (50\%), 'cramping' (50\%), 'pulling' (50\%), 'tender' (66.7\%) and, lastly, 'annoying' (83.3\%). Selection of these descriptors by the patients indicates that the pain experienced is mostly pain with characteristics of pain with neuropathic origin..$^{19}$ These patients seemed to suffer from allodynia. This became evident when reviewing results from the brief questionnaire about pain where it was found that everyday stimuli such as light touch (e.g. with clothing), pressure (e.g. with a finger), and coldness and/or warmth (e.g. from bathing) could elicit pain or worsen pain. Similar observations were reported by Pellkofer et al., where patients had significant heat hyperalgesia and showed a strong tendency towards pain sensitivity for pin-prick and light touch with brush stroking. ${ }^{12}$ However, it should be noted that, for the present study, merely half of the patients experienced this hyper-responsiveness to non-painful or painful stimuli, but not in the craniofacial region. Thermal hyperalgesia is usually attributed to peripheral sensitisation of afferent C-fibres, which typically is a sign of inflammatory processes. ${ }^{17}$ However, Pellkofer et al. found that the presence of hyper-responsiveness to thermal stimuli, especially in response to noxious heat, highly depended on the presence of spinal lesions and that hyperalgesia was particularly severe after a NMOSD relapse, which causes inflammatory demyelination of the spinal cord. 12,20,21 It has been proposed that the degree of thermal hyperalgesia corresponds to the current level of NMOSD-associated neuro-inflammation. ${ }^{12}$ This could explain the observations from this study and why some patients experience hyperalgesia, and others do not. Moreover, this study cannot rule out interference with analgesic treatment and therefore it remains unknown if pain and hypo-/ hyperphenomena could have been more prevalent or more severe without having analgesics on board.
To further investigate the impact of pain in the patients, pain-related interferences were assessed with the SF-BPI. This section of the questionnaire consists of seven items of everyday tasks and situations. Pain was found to correlate with pain-related interferences, at least significantly in the items 'general activity' and 'ability to walk'. Strong correlations were also found between pain and 'mood', 'normal work', 'relations with other people', and 'sleep', which indicates that pain indeed affects patients' everyday life, at least 24 hours prior to the interview. This finding is in accordance with previous studies also showing a correlation between pain and pain-related interferences, ${ }^{1,11,13}$ including sleep ${ }^{15}$ and fatigue. ${ }^{16}$ It should be noted that for each of the items, almost all scores were below 2 on a scale ranging from 0-10. In particular, the items 'ability to walk' and 'relations with other people' were scored quite low (0.8), therefore, it can be questioned whether or not, at least for these two correlations, this is noteworthy information. Also, for the item 'ability to walk', scores were only obtained from five patients, as one patient did not want to rate because she was paralysed from her chest to her feet. Nonetheless, as half of the patients reported pain at the time of interview or within the 3 months preceding the interview, the data from this study emphasise the relatively high incidence of pain in NMOSD patients, which in turn accentuates the need for further investigation.

\section{Pattern of headaches and craniofacial pain}

While little is known about general pattern of pain in NMOSD patients, prevalence and pattern of headache and facial pain are highly underreported in this group of patients. ${ }^{22}$ Some studies have, however, attempted to examine headaches and facial pain; nevertheless, results are limited and indistinct and the type of headache and implicated pathophysiology of pain largely vary. The prevalence of headaches or craniofacial pain observed in this study was not astoundingly high; however, it was noteworthy. Since patient journals (including imaging of CNS) were not assessed in this study, the observations from this interview cannot be linked to clinical findings in the patients. The localisation of lesions in the CNS seems to have a great impact. ${ }^{22,23}$ The prevalence of headaches is higher in patients with medulla oblongata lesions than those without lesions in this area. ${ }^{23}$ In another study exploring the prevalence of brainstem symptoms in NMOSD patients, it was suggested that pain in the facial region and headache might occur as a result of trigeminal neuralgia. Consequently, as no assessment of CNS imaging was made, no strong conclusion regarding the cause of headache or craniofacial pain can be made, at least not at present; but more investigations would facilitate a better understanding of potential underlying mechanisms.

Two of the three patients also reported periodic pain in the orbital region, often occurring after physical exertion. This observation is close to that of Qian et al., who reported a rather high prevalence of retro-orbital pain (55.2\%). ${ }^{11}$ Pain in the orbital regions is often caused by ON. ${ }^{4,22}$ The present study cannot conclude whether or not ON attacks may explain the orbital pain pattern observed. Furthermore, the patients did not report that they suffered from ON. This could have been clarified if patients' full medical journals had been accessible. All three patients experiencing headaches, orbital pain and neck pain were women. Kim et al. found a significant gender difference in regards to site of attack and severity of symptoms and women presented more frequently with relapses. ${ }^{24}$ In general, there is a female preponderance in NMOSD cases. ${ }^{6}$ Since both the sample size and proportion of male patients in this study are small, it is not possible to conclude whether gender effects cause these observations.

Considering some limitations, overall, this study revealed that headaches do occur frequently and are mild to severe in intensity. Additionally, despite a relatively small sample size and a skewed 
gender distribution, this study demonstrated that pain occurs in areas that are frequently overlooked by other pain-related investigations in NMOSD. Furthermore, these findings highlight that headaches and craniofacial pain do occur frequently in this group of patients, much more prevalently than first appreciated. This also highlights the value and necessity of further investigations.

\section{Illness perception}

The BIPQ is a model that allows quick assessment of the illness perception of patients suffering from diseases or illnesses, and the higher the score, the worse the perception of one's illness. ${ }^{18}$ The present study demonstrated that, with a mean score of 38 out of 90 , patients think negatively about their disease, at least to some noteworthy degree. One item of the questionnaire scored exceptionally high for all patients, the 'timeline' item. With a score of 9 out of 10 on the scale, it indicates that patients feel they remain sick for a long time. These findings are partially in accordance with existing literature. Similar results have been found by Zhao et al. and Qian et al., ${ }^{11,13}$ who explored NMOSD patients' evaluation of their own health status through the Short-form Health Survey and found negative evaluations of own health status in these patients. To the best of the authors' knowledge, the present study is the first to report illness perception using the BIPQ in NMOSD patients.

Research has demonstrated the importance of illness representations to patient behavior. ${ }^{25}$ Changing illness perception has been found to improve disease recovery in various illnesses and diseases, and consequently this has helped patients cope better with their disease. ${ }^{26}$ Therefore, the findings of this study may assist in better understanding of patients' own perception on their diagnosis, their feelings, and their concerns. This in turn may facilitate the way that clinicians can help patients to cope with their illness.

\section{Fatigue, anxiety and depression}

NMOSD is associated with reduced quality of life due to the fact that some factors such as fatigue, anxiety, and depression have been known to impact the patients negatively and to a large extent. ${ }^{10,27}$ Fatigue significantly lowers quality of life ${ }^{27-29}$ and it is present in many neurological disorders including NMOSD. ${ }^{16}$ Similarly, patients in the present study were found to suffer from fatigue. This observation is interesting as fatigue is a common comorbidity of NMOSD and it has been proposed that this factor may also enhance the perception of pain. ${ }^{9}$ Additionally, the interrelationship between fatigue and pain can be a vicious cycle for some patients. This becomes evident when reviewing individual results. Intriguingly, the same patients who scored highly on fatigue questionnaires were also the patients who reported frequent pain. Observations from this study accentuate the importance of considering comorbidities, such as fatigue and sleep disturbances ${ }^{15}$ in NMOSD patients. Further research is needed to highlight this situation, and find ways to overcome fatigue as a strategy to reduce pain and suffering in these patients.

Anxiety and depression are highly prevalent in NMOSD. ${ }^{4,10}$ Contradictorily, this study found that anxiety and depression did not affect patients, at least not to a high degree, which is similar to what has been concluded in other studies. ${ }^{1,4,1,13}$ The BDI-FS did, however, reveal a tendency of minimal depression in the patients. This result is still not close to the outcomes of other studies on this point. ${ }^{1,4,11,13}$ It should be highlighted that the small sample size might have influenced the outcome here, as for both BDI-FS and HADS, scores were computed for only five patients. Further investigations are warranted to demonstrate whether a relationship exists between anxiety and depression and pain, and to what extent. This is of a great value, because the co-existence of pain with depression and anxiety is well known ${ }^{30-32}$ and targeting either condition would improve the overall quality of life of affected patients.

\section{Limitations, challenges and future perspectives}

This study is not exempt from limitations. The study size was rather small. This stems from the fact that NMOSD is a rare autoimmune disease and recruiting sufficient numbers of patients, who can meet the inclusion criteria, is a difficult and time-consuming task. A recent study ${ }^{33}$ has looked into the incidence of NMOSD in the central Denmark region and has reported a considerably low incidence rate. The estimated incidence of NMO (2006 criteria) and NMOSD (2015 criteria) were reported to be 0.08 and 0.12 per 100,000 person-years, respectively.33

In addition, a lot of demographic information was missing. This was due to the fact that the correspondence and application process for obtaining permissions from the Data Protection Agency to access the patient's full medical journal requires sufficient time and resources. Hence, only a limited amount of information could be obtained and used in this pilot study.

The study was conducted by a telephone interview as a consequence of the distances between the investigator and the patients. It would, however, have been more advantageous to interview patients face-toface. This style would potentially avoid misunderstandings that might occur during a telephone interview and would have given patients more trust in the interviewer. However, one should consider that face-to-face meeting is more time-consuming and would require flexibility from both the investigator and the patient to meet at a suitable time and location.

The interview was designed as a qualitative structured interview. This means that patients were asked a specific question, to which they could answer with pre-defined answers only; they could not answer a question freely. This was highlighted by patients themselves as an issue, stating that sometimes it was felt that the pre-defined answers did not address the question well enough, and having the opportunity to answer freely would have given a better answer. It should be noted that patients were indeed given the opportunity to address important issues if they wanted to share them. The structured interview design was chosen for this study because several questionnaires were considered to be used in order to thoroughly investigate pain and related issues. To accommodate the needs of patients for sharing important issues, it is proposed that both a structured (questionnaires) and an unstructured (free talk) design should be considered.

The interview was designed in such a way to last a maximum of one hour; however, this could not be adhered to in the actual interview. The investigator ran some small pilot interviews on healthy subjects to ensure that the interview did not exceed one hour. No pilot interview exceeded one hour. It is the investigator's opinion that the actual patient interviews exceeded this one-hour time frame because the patients wanted to share more information than just answering the structured questions. This also highlights the aforementioned challenge. Almost all patients were very interested in sharing their experiences, simply because they desired to help. This should be taken into account in further investigations.

The questionnaires utilised in this study were several, and were time consuming to fill out. Additionally, many of the questionnaires were quite similar to each other. This was especially true for the FSMC and MFIS, the BDI-FS and HADS. Some of the questionnaires regarding pain were 
also quite alike; however, the investigator ensured that questions did not repeat throughout the interview. For the fatigue, anxiety and depression questionnaires, this approach was not possible due to different scoring systems applied on the questionnaires. In future, it may not be necessary to repeat all questionnaires, but merely one questionnaire for fatigue, and one for anxiety and depression. Another approach could be to design a distinct questionnaire, including all aspects of pain, illness perception, fatigue, anxiety and depression. In doing so, redundancy must be avoided to lead to an easy and speedy process of interviews. However, it should be considered that such a custom-made questionnaire developed from the merging of several existing questionnaires would firstly need a validation process. Questionnaires that are valid are preferable due to their high repeatability. ${ }^{31}$

Since headache and craniofacial pain in this group of patients are still under-recognised, and subsequently have not been studied systematically, we believe the findings from this trial would still attract attention and stimulate further research. Choi et al. highly emphasise this in a case report of a young man suffering from intractable headaches. ${ }^{34}$ This young man suffered from headache with radiation to the posterior neck for several weeks. He was initially mistakenly diagnosed with cervicogenic headache and was treated with a nerve block in the occipital, which proved unsuccessful in relieving the headache..$^{34}$ Further investigation of the patient and his clinical presentations finally led to a NMOSD diagnosis. ${ }^{34}$ Therefore, it is essential to take investigations further in order for scientists and clinicians to know more about the pattern of headaches or craniofacial pain in NMOSD. This knowledge may also prove useful in identifying distinct clinical presentations, or a proper and faster treatment choice.

\section{Conclusion}

This study demonstrated that headaches and some orbital pain occur in an episodic to frequent fashion, and that pain in other parts of the patient's body seems to occur in a frequent to constant manner. More investigation is warranted to explore this finding as this in turn may contribute to better understanding of the underlying mechanisms of not only pain generation in general, but also headaches and craniofacial pain in this group of patients. This will eventually open up an avenue for better management of pain in NMOSD patients.
1. Kanamori Y, Nakashima I, Takai Y, et al. Pain in neuromyelitis optica and its effect on quality of life: a cross-sectional study
Neurology. 2011;77:652-8.

2. Roman-Filip C, Ungureanu A and Cernusca-Mitaru M. Painful tonic spasms and brainstem involvement in a patient with neuromyelitis optica spectrum disorder. Neurol Neurochir Pol. 2016;50:55-8

3. Trebst $\mathrm{C}$, Jarius $\mathrm{S}$, Berthele $\mathrm{A}$, et al. Update on the diagnosis and treatment of neuromyelitis optica: recommendations of the Neuromyelitis Optica Study Group (NEMOS). J Neurol, 2014;261:1-16. DOI: 10.1007/s00415-013-7169-7.

4. Mutch K, Methley A, Moore P, Jacob A. Life on hold: the experience of living with neuromyelitis optica. Disabil Rehabi. 2014;36:1100-7

5. Pandit L, Asgari N, Apiwattanakul $\mathrm{M}$, et al. Demographic and clinical features of neuromyelitis optica: a review. Mult Scler. 2015;21:845-53.

6. Jarius S, Wildemann B, Paul F. Neuromyelitis optica: clinica features, immunopathogenesis and treatment. Clin Exp Immunol. 2014:176:149-64.

7. Bradl M, Kanamori Y, Nakashima I, et al. Pain in neuromyelitis optica--prevalence, pathogenesis and therapy. Nat Rev Neurol. 2014;10:529-36

8. Wingerchuk DM, Banwell B, Bennett JL, et al. International consensus diagnostic criteria for neuromyelitis optica spectrum disorders. Neurology. 2015;85:177-89.

9. Kremer L, Mealy M, Jacob A, et al. Brainstem manifestations in neuromyelitis optica: a multicenter study of 258 patients. Mult scler. 2014;20:843-7.

10. Chavarro VS, Mealy MA, Simpson A, et al. Insufficient treatment of severe depression in neuromyelitis optica spectrum disorder. Neurol Neuroimmunol Neuroinflamm. 2016;3:e286.

11. Qian P, Lancia S, Alvarez E, et al. Association of neuromyelitis optica with severe and intractable pain. Arch Neurol. 2012;69:1482-7.
12. Pellkofer HL, Havla J, Hauer D, et al. The major brain endocannabinoid 2-AG controls neuropathic pain and mechanical hyperalgesia in patients with neuromyelitis optica. mechanical hyperalgesia

13. Zhao $S$, Mutch $K$, Elsone $L$, et al. Neuropathic pain in neuromyelitis optica affects activities of daily living and quality of life. Mult Scler. 2014;20:1658-6

14. Moloney N, Wrigley P and Hush J. Somatosensory assessment in chronic pain: progress and potential. Pain Manag. 2016;6:125-8.

15. Song Y, Pan L, Fu Y, et al. Sleep abnormality in neuromyelitis optica spectrum disorder. Neurol Neuroimmunol Neuroinflamm. 2015;2:e94.

16. Penner IK, Paul F. Fatigue as a symptom or comorbidity of neurological diseases. Nat Rev Neurol. 2017;13:662-75.

17. Simone DA. Neural mechanisms of hyperalgesia. Curr Opin Neurobiol. 1992;2:479-83.

18. Broadbent E, Petrie KJ, Main J, Weinman J. The Brief Illness Perception Questionnaire. J Psychosom Res. 2006;60:631-7.

19. Fear C. Neuropathic pain: clinical features, assessment and treatment. Nurs Stand. 2010;25:35-40.

20. Treede RD, Meyer RA, Raja SN, Campbell JN. Periphera and central mechanisms of cutaneous hyperalgesia. Prog Neurobiol. 1992;38:397-421.

21. Saadoun $S$, Waters $P$, Bell BA, et al. Intra-cerebral injection of neuromyelitis optica immunoglobulin $\mathrm{G}$ and human complement produces neuromyelitis optica lesions in mice. Brain. 2010;133:349-61.

22. Masters-Israilov $A$, Robbins MS. Headache in Neuromyelitis Optica. Curr Pain Headache Rep. 2017;21:20.

23. Wang $Y Q$, Zhang $L$, Zhang BJ, et al. Comparative clinical characteristics of neuromyelitis optica spectrum disorders with and without medulla oblongata lesions. J Neurol. 2014:261:954-62.

24. Kim SMM, Waters $P$, Woodhall M, et al. Gender effect on neuromyelitis optica spectrum disorder with aquaporin4mmunoglobulin G. Mult Scler I. 2017:23:1104-11.

25. Kucukarslan SN. A review of published studies of patients illness perceptions and medication adherence: lessons learned and future directions. Res Social Adm Pharm. 2012;8:371-82.

26. Petrie KJ, Jago LA, Devcich DA. The role of illness perceptions in patients with medical conditions. Curr Opin Psychiatr. 2007;20:163-7

27. Shi ZY, Chen HX, Lian ZY, et al. Factors that impact healthrelated quality of life in neuromyelitis optica spectrum disorder: anxiety, disability, fatigue and depression. J Neuroimmunol. 2016;293:54-8.

28. Hyun JW, Kim SH, Jeong IH, et al. Increased frequency and severity of restless legs syndrome in patients with neuromyelitis optica spectrum disorder. Sleep Med. 2016:17:121-3.

29. Akaishi T, Nakashima I, Misu T, et al. Depressive state and chronic fatigue in multiple sclerosis and neuromyelitis optica. I Neuroimmunol. 2015;283:70-3.

30. Radat F, Margot-Duclot A, Attal N. Psychiatric co-morbidities in patients with chronic peripheral neuropathic pain: a multicentre cohort study. Eur J Pain. 2013;17:1547-57.

31. Castro M, Kraychete D, Daltro C, et al. Comorbid anxiety and depression disorders in patients with chronic pain. Arq Neuropsiquiatr. 2009;67:982-5.

32. $\mathrm{Ko} \mathrm{HJ}$, Seo SJ, Youn $\mathrm{CH}$, et al. The association between pain and depression, anxiety, and cognitive function among advanced cancer patients in the hospice ward. Korean I Fam Med. 2013;34:347-56

33. Dale GH, Svendsen KB, Gjelstrup MC, et al. Incidence of neuromyelitis optica spectrum disorder in the Central Denmark Region. Acta Neurol Scand. 2018;137:582-88.

34. Choi SI, Lee YJ, Kim DW, Yang JY. A case of neuromyelitis optica misdiagnosed as cervicogenic headache. Korean J Pain. 2014;27:77-80. 